EPJ manuscript No.

(will be inserted by the editor)

\title{
Constraints on the time-scale of nuclear breakup from thermal hard-photon emission
}

\author{
R. Ortega ${ }^{1,2 a}$, D. d'Enterria ${ }^{1,2,3 b}$, G. Martínez ${ }^{2,3}$, D. Baiborodin ${ }^{4}$, H. Delagrange ${ }^{2,3}$, J. Díaz $^{5}$, F. Fernández ${ }^{1}$ \\ H. Löhner ${ }^{6}$, T. Matulewicz ${ }^{7}$, R.W. Ostendorf ${ }^{6}$, S. Schadmand ${ }^{8}$, Y. Schutz ${ }^{2,3 c}$, P. Tlusty ${ }^{4}$, R. Turrisi ${ }^{3 d}$, V. Wagner ${ }^{4}$, \\ H.W. Wilschut ${ }^{6}$, and N. Yahlali ${ }^{5}$ \\ 1 Grup de Física de les Radiacions, Universitat Autònoma de Barcelona 08193, Catalonia \\ 2 SUBATECH, 4, rue Alfred Kastler BP20722 44307 Nantes Cedex 3, France \\ 3 GANIL, Grand Accélérateur National d'Ions Lourds, IN2P3-CNRS, DSM-CEA, 14076 Caen Cedex 5, France \\ 4 Institute of Nuclear Physics, 20568 Rez̃, Czech Republic \\ 5 Institut de Física Corpuscular, Universitat de Valencia-CSIC, Dr Moliner 50, 46100 Burjassot, Spain \\ 6 Kernfysisch Versneller Instituut, 9747 AA Groningen, The Netherlands \\ 7 Institut of Experimental Physics, Warsaw University, 00681 Warsaw, Poland \\ 8 Institut für Kernphysik, Forschungszentrum Jülich, D-52457 Jülich, Germany
}

Received: date / Revised version: date

\begin{abstract}
Measured hard photon multiplicities from second-chance nucleon-nucleon collisions are used in combination with a kinetic thermal model, to estimate the break-up times of excited nuclear systems produced in nucleus-nucleus reactions at intermediate energies. The obtained nuclear break-up time for the ${ }^{129} \mathrm{Xe}+{ }^{n a t} \mathrm{Sn}$ reaction at $50 A \mathrm{MeV}$ is $\Delta \tau \approx 100-300 \mathrm{fm} / c$ for all reaction centralities. The lifetime of the radiating sources produced in seven other different heavy-ion reactions studied by the TAPS experiment are consistent with $\Delta \tau \approx 100 \mathrm{fm} / c$, such relatively long thermal photon emission times do not support the interpretation of nuclear breakup as due to a fast spinodal process for the heavy nuclear systems studied.
\end{abstract}

PACS. 21.65.+f - 25.70.-z - 13.75.Cs $-64.70 . \mathrm{Dv}$

\section{Introduction}

Nucleus-nucleus collisions are the only available tool to explore in the laboratory the different domains of the nuclear phase diagram. In heavy-ion (HI) collisions at intermediate energies (bombarding energies between $20 \mathrm{~A}$ $\mathrm{MeV}$ and $100 \mathrm{~A} \mathrm{MeV}$ ) the projectile and target traverse each other in a time scale $t \lesssim 50 \mathrm{fm} / c$ forming a compressed and excited transient system that usually disassembles into several intermediate-mass-fragments (IMF) in a process known as "nuclear multifragmentation" 1 . The exact physical mechanism that drives this breakup is up to date an open issue. Two opposite scenarios have been considered: a fast breakup from an expanding source, consistent with a spontaneous spinodal mechanism, and a sequential slower breakup from a thermally equilibrated source. The knowledge of the relative importance of these

\footnotetext{
a Present address: Physikalisches Institut, Universität Heidelberg, Philosophenweg 12 D-69120 Heidelberg, Germany

b Present address: Nevis Laboratories, Columbia University 538 W. 120th St. NY 10027, USA

c Present address: CERN, CH-1211 Genève, Switzerland

d Present address: INFN-Padova, Via Marzolo 8, 35131 Padova, Italy
}

two mechanisms is essential to find-out a possible connection between nuclear multifragmentation and the occurrence of a "liquid-gas"-like phase transition [2 in nuclear systems with excitation energies in the range $\epsilon^{\star}=(3-8) A$ $\mathrm{MeV}$, where a flattening of the "caloric curve", signaling a possible liquid-gas phase transition, has been observed 3 , 4 4, 5, 6. To discern between both possible mechanisms it is necessary to establish the breakup time of the multifragmenting system.

The experimental observable used in the present analysis in order to probe the thermodynamical state of the produced transient nuclear systems, and in particular, to delimit the nuclear breakup time is thermal hard photon emission. At intermediate-energy HI collisions, photons above $30 \mathrm{MeV}$ (hard photons) issue from bremsstrahlung in incoherent proton-neutron collisions [7. The main hard photon contribution, the so-called "direct" component, is emitted from first-chance nucleon-nucleon $(N N)$ collisions in the preequilibrium stage of the reaction. A second and softer flash of hard photons is emitted from secondary $N N$ collisions in the later stages 8,9 from a thermalizing source 10. Hence, the experimental hard photon spectrum measured in HI reactions has been found to be well reproduced by the sum of two exponential distributions 
[9]:

$$
\frac{d \sigma}{d E_{\gamma}}=K_{d} e^{-E_{\gamma} / E_{0}^{d}}+K_{t} e^{-E_{\gamma} / E_{0}^{t}}
$$

where $d$ and $t$ stand for direct and thermal, respectively, and the factors $K_{d, t}$ are related to the intensity of each source. The power of first chance bremsstrahlung photons is the fact that they result from incoherent collisions. This allowed one to equate the observed intensity and slope of the gamma spectrum with the number and hardness of collisions respectively. With the kinematic thermal model 11] this information can also be obtained from secondary collisions; the soft spectrum now corresponds to the number of collisions integrated over the lifetime of the equilibrated source and its formation, and the slope parameter is related to the average temperature of the source [12. Although multiple collisions lead to bremsstrahlung quenching (see e.g. 13]) it is believed not to play an important role because we exploit the photon spectrum above $E_{\gamma c u t}=30 \mathrm{MeV}$. To observe about $10 \%$ quenching the collision rate of the nucleons in the thermal source should not exceed $E_{\gamma c u t} / \mathrm{hbar}=1.5 \cdot 10^{-1}(\mathrm{fm} / \mathrm{c})^{-1}$, in the model, without considering Pauli blocking, the rate typically is $1.3 \cdot 10^{-1}(\mathrm{fm} / \mathrm{c})^{-1}$. Thus the time scales derived in this work are not limited by the quenching phenomena.

The analysis presented in this paper has two different goals. First, we want to elucidate the dependence of the thermal photon temperature on the reaction centrality in ${ }^{129} \mathrm{Xe}+{ }^{n a t} \mathrm{Sn}$ reactions at $50 \mathrm{AMeV}$; at this energy, this heavy and symmetric system provides good conditions for observing the effects of a possible nuclear liquid-gas phase transition, and moreover, the charged-particle distributions and related observables have been studied in detail for this reaction by the INDRA Collaboration (see e.g. 14. [15, 16, 17, 18 and references therein). The second objective consists in assessing the nuclear breakup time-scales using the measured second-chance photon multiplicities and a thermal model that describes well the observed spectra. This investigation is performed for 7 different reactions studied by the TAPS Collaboration: ${ }^{86} \mathrm{Kr}+{ }^{58} \mathrm{Ni}$ at $60 \mathrm{~A}$ $\mathrm{MeV}\left[8,{ }^{181} \mathrm{Ta}+{ }^{197} \mathrm{Au}\right.$ at $40 \mathrm{~A} \mathrm{MeV}\left[8,{ }^{208} \mathrm{~Pb}+{ }^{197} \mathrm{Au}\right.$ at $30 A \mathrm{MeV}$ [8, ${ }^{36} \mathrm{Ar}+{ }^{107} \mathrm{Ag}$ at $60 A \mathrm{MeV}$ 10, ${ }^{36} \mathrm{Ar}+$ ${ }^{197} \mathrm{Au}$ at $60 A \mathrm{MeV}\left[10,{ }^{36} \mathrm{Ar}+{ }^{58} \mathrm{Ni}\right.$ at $60 A \mathrm{MeV}$ [10 and ${ }^{129} \mathrm{Xe}+{ }^{n a t} \mathrm{Sn}$ at $50 \mathrm{~A} \mathrm{MeV}$. The paper is organized as follows: in Sect. 2 the experimental setup is described. In Sect. [3 the particle identification methods are presented. The results obtained from the analysis of the inclusive hard photon spectrum are summarized in Sect. [4 In Sect. 5 we focus on the $\mathrm{Xe}+{ }^{n a t} \mathrm{Sn}$ exclusive measurements; first, we present the experimental centrality classes as well as the method employed to estimate the average impact parameter; next, we present the direct and thermal hard photon measurements for each centrality class. In Sect. 6. the thermodynamical properties extracted from the thermal hard photon signal are presented. In particular in 6.2 the results on the lifetimes of the thermal hard photon sources obtained for the different reactions are presented. Section $\mathbf{7}$ gives a summary.

\section{Experimental setup}

The experiment E-300 was performed at the "Grand Accélérateur National d'Ions Lourds" (GANIL) located in Caen, France, during 13 days of total beam time in May 1998. The GANIL accelerator system delivered a $\mathrm{Xe}^{46+}$ beam of $50 \mathrm{~A} \mathrm{MeV}$ with a bunch rate of $10 \mathrm{MHz}$ and a nominal intensity of $5 \mathrm{nA}$, around 68 ions per pulse. The Xe ions impinged on a ${ }^{\text {nat }} \mathrm{Sn}$ target of $1 \mathrm{mg} / \mathrm{cm}^{2}$ thickness (with associated interaction probability of $1.9 \cdot 10^{-3}$ per burst). The experimental setup consisted of the "Two Arms Photon Spectrometer" TAPS associated with three particle multidetectors: the GANIL Silicon Strip Detector (SSD), the Washington University "Dwarf Ball" (DB) and the KVI "Forward Wall" (FW). A general layout of the experimental setup is presented in Fig. [1]

The TAPS electromagnetic calorimeter [19] consists of 384 scintillation modules. Each one consisted of a $\mathrm{BaF}_{2}$ hexagonal crystal, $2.95 \mathrm{~cm}$ inner radius and $25 \mathrm{~cm}$ length (corresponding to $12 X_{0}$ radiation lengths) [20,21, and a Charged Particle Veto (CPV) detector of plastic (NE102A) scintillator. TAPS offers a large solid angle coverage allowing for high quality angular distribution measurements in a wide energy range, from statistical $\left(\mathrm{E}_{\gamma}>5 \mathrm{MeV}\right)$ to the hardest photons $\left(\mathrm{E}_{\gamma} \approx 200 \mathrm{MeV}\right)$. In this experiment TAPS modules were arranged in six blocks of 64 modules $(8 \times 8)$, which were placed in an almost symmetrical configuration in the horizontal plane around the target, and at an average distance of $56 \mathrm{~cm}$, covering about $20 \%$ of the full solid angle.

The GANIL silicon strip detector telescope SSD was placed at $19 \mathrm{~cm}$ downstream the target inside the vacuum chamber, covering the angular range of $2.2^{\circ} \leq \theta \leq 10.3^{\circ}$. This device, consisting of a first disk $150 \mu \mathrm{m}$ thick with 64 semicircular strips and of a second disk $500 \mu \mathrm{m}$ thick with 128 radial strips, was sensitive to projectile-like fragments (PLF) emitted in binary reactions and intermediate-mass fragments (IMF) emitted in the forward direction from more dissipative reactions.

The Washington University "Dwarf Ball" (DB) 22 covered an angular range of $31^{\circ} \leq \theta \leq 168^{\circ}$ around the target. This detector system allows to identify lightcharged particles (LCP) and IMF. The Dwarf Ball (DB) consists of 64 phoswich detectors forming a sphere with an inner radius of $41.5 \mathrm{~mm}$. Each phoswich detector is made of a thin plastic scintillator and of a $\mathrm{CsI}(\mathrm{Tl})$ inorganic crystal scintillator. The plastic scintillator type (Bicron BC400 or BC446) depends on the covered angle and so does its thickness, $10 \mu \mathrm{m}$ and $40 \mu \mathrm{m}$ for the most backward and forward modules, respectively. The $\mathrm{CsI}(\mathrm{Tl})$ crystal glued on the back of each plastic is $4 \mathrm{~mm}$ to $8 \mathrm{~mm}$ thick, depending also on the angle.

The KVI "Forward Wall" $(\mathrm{FW})$ 23, consisted of 92 plastic scintillator phoswiches, detects and identifies LCP and IMF (up to $\mathrm{Z} \approx 15$ ) emitted in the forward direction. In our experimental setup, this detector was placed downstream $74 \mathrm{~cm}$ away from the target covering the forward hemisphere behind the SSD, $2.5^{\circ} \leq \theta \leq 25^{\circ}$ and the whole azimuthal range. Each $\mathrm{FW}$ phoswich is composed of two organic plastic scintillators which are heat-pressed 


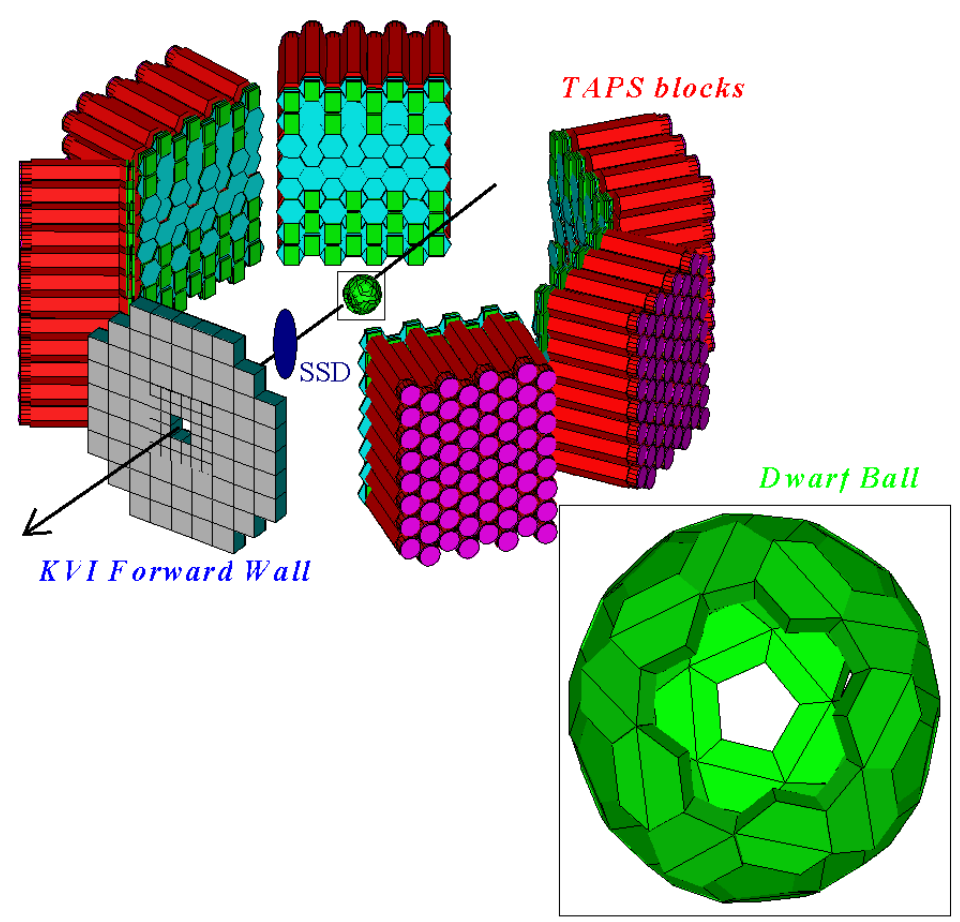

Fig. 1. General layout of the experimental setup used to study the reaction ${ }^{129}{ }^{X e+}+{ }^{\text {nat }}$ Sn at $50 A$ MeV at GANIL. The detector system consists of the Silicon Strip Detector (SSD), the Washington University Dwarf Ball (DB), the TAPS electromagnetic calorimeter and the KVI Forward Wall $(F W)$.

together; a $1 \mathrm{~mm}$ thick "fast" ( $\tau=2.4 \mathrm{~ns})$ NE102A plastic followed by a $50 \mathrm{~mm}$ thick "slow" ( $\tau=320 \mathrm{~ns})$ NE115 plastic.

This complete experimental setup allowed correlated measurements of photons and charged-particles, necessary to study the dependence of the hard-photon production on the reaction topology. The photon data were recorded with the minimum bias trigger for neutral particles $\left(\gamma^{*} \mathrm{DB}\right)$, which signaled events with at least one neutral hit in TAPS of energy $E_{\gamma}>10 \mathrm{MeV}$ detected in coincidence with one or more charged particles in the Dwarf Ball. The minimum bias $(\mathrm{MB})$ reaction trigger was defined by the condition of detecting at least a charged particle in the Dwarf Ball.

\section{Particle identification}

Photons are identified in TAPS by means of a Pulse Shape (PSA) vs. time-of-flight (TOF) analysis and the CPV information. Photons and leptons induce a stronger intensity of the fast $\mathrm{BaF}_{2}$ light component compared to hadronic particles, producing therefore a higher ratio of the fast to the slow energy components in the $\mathrm{BaF}_{2}$ (PSA). Photons and relativistic electrons are located in time within a prompt peak centered at $\mathrm{TOF}=1.87 \mathrm{~ns}$ (with a resolution $\sigma=340$ ps 24 ); these particles produce hence a recognizable contour in the PSA-TOF spectrum. In order to separate photons from relativistic electrons the information (fired or not) delivered by the CPV of each module is used. Nevertheless, given that cosmic muons have mainly vertical trajectories not firing the CPV, and have random TOF, they can be misidentified as photons. This is avoided by defining a PSA-TOF cosmic contour several nanoseconds far from the photon contour but with the same dimensions. Subsequently, the energy and angular spectra measured within this contour (see Fig. 2) are subtracted from the raw photon energy and angular spectra respectively.

The $\Delta \mathrm{E}$ - E telescope technique 24,25$]$ allows the charge identification of LCP and IMF detected in the DB and in the FW, as well as of the PLF and IMF impinging on the SSD. The light measured in the first stage of each multidetector (the first component of each phoswich in the DB and the FW, or the first disk in the SSD) and the signal of the second component are plotted in bidimensional histograms in which the different curves correspond to different $\mathrm{Z}$. In the DB, additional LCP isotopic identification was also possible exploiting the pulse shape properties of the $\mathrm{CsI}(\mathrm{Tl})$ light.

\section{Inclusive hard photon spectra in ${ }^{129} \mathrm{Xe}+$ ${ }^{n a t} \mathrm{Sn}$ at $\mathbf{5 0} A \mathrm{MeV}$ reactions}

Figure 2 shows the inclusive raw photon energy spectrum measured in the ${ }^{129} \mathrm{Xe}+{ }^{n a t} \mathrm{Sn}$ reaction from the collection 


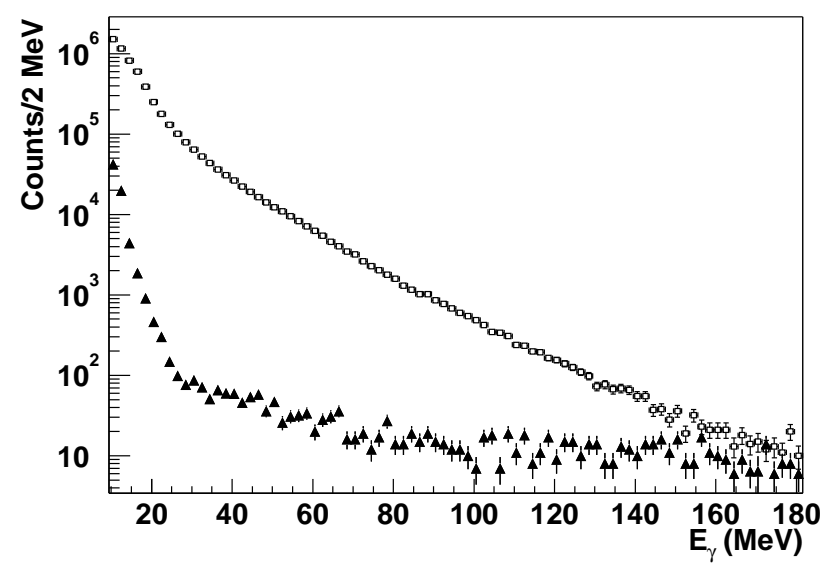

Fig. 2. Inclusive raw photon spectrum measured in the centerof-mass frame for the reaction ${ }^{129} \mathrm{Xe}+{ }^{n a t} \mathrm{Sn}$ at $50 \mathrm{~A} \mathrm{MeV}$. The triangles show the cosmic ray background contribution.

of $5.8 \cdot 10^{6}$ photons with $E_{\gamma}>10 \mathrm{MeV}$ detected in TAPS and transformed into the $\mathrm{CM}$ frame $\left(\beta_{A A} \approx \beta_{N N} \approx 0.15\right)$. After cosmic-ray subtraction, and for energies $E_{\gamma}>30$ $\mathrm{MeV}$ this spectrum is well described by the double source fit of Eq. [1(Fig. 3). The direct component exhibits an inverse slope parameter $E_{0}^{d}=(15.6 \pm 1.0) \mathrm{MeV}$ whereas the thermal slope parameter is $E_{0}^{t}=(7.0 \pm 0.6) \mathrm{MeV}$. Both slopes are in agreement with the direct [26] and thermal 12] photon slope systematics measured in intermediate $A+A$ reactions. Thermal hard photons account for $22 \%$ of the total yield above $30 \mathrm{MeV}$. The strength and intensity of the thermal emission are also confirmed by a double source analysis of the hard photon angular distribution; however, due to the symmetry of the system, the $\mathrm{NN}$ and AA source velocities cannot be disentangled [27].

The direct (thermal) hard photon multiplicity, i.e. the number of direct (thermal) hard photons emitted per nuclear reaction, has been obtained via the following expression:

$$
M_{\gamma}^{i}=\frac{\sigma_{\gamma}^{i}}{\sigma_{R}}
$$

where $i$ stands for direct or thermal, $\sigma_{\gamma}^{i}$ is the experimental hard photon cross section determined by integrating the corresponding $d \sigma / d E_{\gamma}$ spectrum above $E_{\gamma}=30 \mathrm{MeV}$, and $\sigma_{R}=(5300 \pm 600) \mathrm{mb}$ is the experimental total reaction cross section for $\mathrm{Xe}+\mathrm{Sn}$, obtained from the charged particle distribution measured in the $\mathrm{DB}$ with the $\mathrm{MB}$ reaction trigger. We report the direct and thermal hard photon cross sections and multiplicities in Table 1 The direct hard photon probability $P_{\gamma}^{d}$, i.e. the probability to produce a hard photon in a first-chance proton-neutron collision, is determined from the experimental direct hard photon multiplicity:

$$
P_{\gamma}^{d}=\frac{\sigma_{\gamma}^{d}}{\sigma_{R} \cdot\left\langle N_{p n}\right\rangle_{b}}=\frac{M_{\gamma}^{d}}{\left\langle N_{p n}\right\rangle_{b}}
$$

Table 1. Summary of the experimental inclusive hard photon $\left(E_{\gamma}>30 \mathrm{MeV}\right)$ results obtained for the ${ }^{129} \mathrm{Xe}+{ }^{\text {nat }} \mathrm{Sn}$ reaction at $50 \mathrm{~A} \mathrm{MeV}$. For the direct and thermal $\gamma$ components we quote: inverse slope $E_{0}$, relative intensity $I$, source velocity $\beta$, cross-section $\sigma_{\gamma}$, multiplicity $M_{\gamma}$, and (only for the direct component) hard photon probability $P_{\gamma}$.

\begin{tabular}{lll}
\hline & Direct & Thermal \\
\hline$E_{0}(\mathrm{MeV})$ & $15.6 \pm 1.0$ & $7.0 \pm 0.6$ \\
$I(\%)$ & $78 \pm 1$ & $22 \pm 1$ \\
$\beta$ & $0.15 \pm 0.01$ & $0.16 \pm 0.01$ \\
$\sigma_{\gamma}(\mathrm{mb})$ & $4.9 \pm 0.6$ & $1.4 \pm 0.2$ \\
$M_{\gamma}$ & $(9.3 \pm 0.8) \cdot 10^{-4}$ & $(2.6 \pm 0.3) \cdot 10^{-4}$ \\
$P_{\gamma}$ & $(7.8 \pm 0.7) \cdot 10^{-5}$ & - \\
\hline
\end{tabular}


Fig. 3. Left panel: experimental inclusive hard photon spectrum measured for the reaction ${ }^{129} \mathrm{Xe}+{ }^{n a t} \mathrm{Sn}$ at $50 \mathrm{~A}$ in the range $E_{\gamma}=30-65 \mathrm{MeV}$, fitted to the Eq. 1, the solid (dashed) line is the direct (thermal) exponential distribution. Right panel: inclusive thermal hard photon spectrum obtained after subtracting the fitted direct component.

where $\left\langle N_{p n}\right\rangle_{b}$ is the number of first chance pn collisions averaged over impact parameter, calculated from the geometrical "equal-participant" model of Nifenecker and Bon$\operatorname{dorf}[28] .\left\langle N_{p n}\right\rangle_{b}$ is $=11.79$ for the Xe on Sn system. The measured $P_{\gamma}^{d}$ in ${ }^{129} \mathrm{Xe}+{ }^{n a t} \mathrm{Sn}$ reactions at $50 \mathrm{~A} \mathrm{MeV}$ is $(7.8 \pm 0.7) \cdot 10^{-5}$. We note that we do not quote a thermal hard photon probability, since we should determine the average number of secondary (and not primary) pn collisions, from which the thermal component originates, and this number is not well determined experimentally nor theoretically. More details of the inclusive analysis can be found in 24]. 
R. Ortega et al.: Constraints on the time-scale of nuclear breakup from thermal hard-photon emission

Table 2. Conditions on the fragment multiplicity in the $S S D\left(M^{S S D}\right)$, the charged particle multiplicity (IMF + LCP) in the $D B\left(M^{D B}\right)$ and the $F W\left(M^{F W}\right)$ that define each centrality class. The contribution to the reaction cross-section, and to the nuclear reactions detected by the minimum bias photon trigger $\left(\gamma^{*} D B\right)$ for each centrality class are reported.

\begin{tabular}{llll}
\hline Centrality class & Multiplicity condition & $\% \sigma_{R}$ & $\% \sigma_{R}\left(\gamma^{*} \mathrm{DB}\right)$ \\
\hline A or Periph. & $M^{D B+F W}=1-2$ and $M^{S S D}(\theta<5)=0$ & $57 \%$ & $23 \%$ \\
B & $M^{D B+F W}=1-2$ & $67 \%$ & $26 \%$ \\
C & $2<M^{D B+F W}<8$ & $38 \%$ & $58 \%$ \\
D & $5<M^{D B+F W}<9$ & $10 \%$ & $24 \%$ \\
E & $6<M^{D B+F W}<10$ & $7 \%$ & $18 \%$ \\
F or Central & $9<M^{D B+F W}<15$ & $2 \%$ & $6 \%$ \\
\hline
\end{tabular}

Table 3. Characteristics of the six centrality classes considered for Xe+Sn reactions at $50 A$ MeV. For each centrality bin we quote: average charged particle multiplicity $M_{c p}$ and impact parameter $\langle b\rangle_{g e o}$ estimated with the geometrical model of Cavata et al. [29]; direct photon multiplicity $\left(M_{\gamma}^{d}\right)$, average number of first chance proton-neutron collisions $\left\langle N_{p n}\right\rangle$ obtained as described in [30 31], and associated averaged impact parameter $\langle b\rangle_{\gamma}$ estimated using the "equal-participant" model [28].

\begin{tabular}{llllll}
\hline Centrality class & $\left\langle M_{c p}\right\rangle$ & $\langle\mathrm{b}\rangle_{g e o}(\mathrm{fm})$ & $M_{\gamma}^{d}$ & $\left\langle N_{p n}\right\rangle$ & $\langle\mathrm{b}\rangle_{\gamma}(\mathrm{fm})$ \\
\hline A or Periph. & 1.5 & $9.4 \pm 0.8$ & $(3.2 \pm 0.2) \cdot 10^{-4}$ & $4.1 \pm 0.6$ & $9.2 \pm 0.3$ \\
B & 1.5 & $9.4 \pm 0.8$ & $(4.1 \pm 0.3) \cdot 10^{-4}$ & $5.2 \pm 0.6$ & $8.8 \pm 0.3$ \\
C & 4.7 & $5.2 \pm 0.4$ & $(1.5 \pm 0.1) \cdot 10^{-3}$ & $18.6 \pm 2.2$ & $5.9 \pm 0.5$ \\
D & 6.8 & $3.3 \pm 0.3$ & $(2.4 \pm 0.2) \cdot 10^{-3}$ & $30.7 \pm 3.7$ & $4.0 \pm 0.6$ \\
E & 7.8 & $2.6 \pm 0.2$ & $(2.8 \pm 0.2) \cdot 10^{-3}$ & $36.1 \pm 4.4$ & $3.1 \pm 0.7$ \\
F or Central & 11.4 & $0.9 \pm 0.1$ & $(3.8 \pm 0.4) \cdot 10^{-3}$ & $48.9 \pm 6.7$ & $1.3 \pm 1.1$ \\
\hline
\end{tabular}

\section{Exclusive hard photon analysis in ${ }^{129} \mathrm{Xe}+$ ${ }^{n a t} \mathrm{Sn}$ at $50 \mathrm{~A} \mathrm{MeV}$ reactions}

\subsection{Selection of centrality classes}

Six centrality classes of ${ }^{129} \mathrm{Xe}+{ }^{n a t} \mathrm{Sn}$ collisions have been selected to investigate the dependence of the thermal hard photon production on the reaction centrality. This choice results from a compromise between requiring a wide impact parameter coverage and obtaining enough hard photon statistics for each centrality class. We report in Table 2 the conditions on the fragment multiplicities measured in the SSD, Dwarf Ball and Forward Wall that define the selected centrality bins.

\subsection{Estimation of the impact parameter for minimum bias data}

In order to estimate the impact parameter $\mathrm{b}$ in ${ }^{129} \mathrm{Xe}+$ ${ }^{n a t} \mathrm{Sn}$ all reactions, recorded by means of the MB trigger, we apply the geometrical method proposed by Cavata et al. 29, which relies on the monotonous decrease of $M_{C P}$ as a function of $b$. An alternative method to estimate the impact parameter in experiments where hard photon emission can be analyzed is based on the correlation of the direct hard $\gamma$ yield with the impact parameter 30 31. In this method, for a given reaction class, the experimental average number of first chance $p n$ collisions $\left\langle N_{p n}\right\rangle$ is estimated, making use of Eq. 3. from the experimentally measured $M_{\gamma}^{d}$ and from the $P_{\gamma}^{d}$, which is considered to depend only on the Coulomb corrected bombarding energy. The obtained $\left\langle N_{p n}\right\rangle$ values are related to the impact parameter b by the geometrical "equal-participant" model of of Nifenecker and Bondorf [28. We note that if the impact parameter is obtained directly from the total hard photon yield, as commonly done, instead of only from the direct component, the deduced impact parameter might be distorted for reactions where a thermal photon contribution cannot be neglected. The average impact parameter for each reaction class, obtained by means of the direct hard photon multiplicities and by means of the charged particle multiplicities measured in the DB, are reported in Table 3. We find a good agreement between both methods, and in addition the whole impact parameter range is covered.

\subsection{Determination of the impact parameter for reactions where a hard photon is produced}

We want to exploit the properties of the thermal hard photon component as a tool to extract the thermodynamical state of the radiating source. It is therefore of crucial importance to determine as accurately as possible any bias introduced in the estimation of the impact parameter for particular reactions where a hard photon is produced.

Within a given centrality class, defined by a range in the $M_{c p}$ distribution, hard photons are mostly emitted in reactions with high $M_{c p}$ values, characterized by a large projectile-target overlap, and consequently by a high number of $p n$ collisions. This bias through lower impact pa- 
rameters can be reproduced by the geometrical "equalparticipant" model; the reaction cross-section as a function of $\mathrm{b}$ for reactions where a hard photon is produced, $\sigma_{R}^{\gamma}(\mathrm{b})$, is modulated by the product of $\sigma_{R}(\mathrm{~b})$ and $N_{p n}(\mathrm{~b})$. As a consequence, the ratio between the reaction crosssection $\sigma_{R}^{\gamma}(\mathrm{b})$ and the total (integrated over b) $\sigma_{R}^{\gamma}$ is a representable function sensitive to the cross section deviation through lower b (see Fig. (4). It is thus possible to estimate the average impact parameter for reactions where a hard photon is produced by means of an extension of the geometrical model of Cavata et al., correlating the experimental $\sigma_{R}^{\gamma}\left(M_{c p}\right) / \sigma_{R}^{\gamma}$ and the theoretical $\sigma_{R}^{\gamma}\left(b, N_{p n}\right) / \sigma_{R}$ obtained from the geometrical "equal-participant" model. The average b of each centrality class has been obtained with this method after weighting the $\mathrm{b}\left(M_{c p}\right)$ by the contribution of each $M_{c p}$ within the centrality class. Table 4 reports the $\langle\mathrm{b}\rangle$ estimated for each $\mathrm{RC}$ for reactions where a hard $\gamma$ is emitted.

Table 4. Estimated average impact parameter $\langle b\rangle$ for each of the six centrality classes considered.

\begin{tabular}{ll}
\hline Centrality class & $\langle\mathrm{b}\rangle_{\text {geo }}(\mathrm{fm})$ \\
\hline Class A or Peripheral & $7.6 \pm 0.3$ \\
Class B & $7.2 \pm 0.3$ \\
Class C & $4.2 \pm 0.4$ \\
Class D & $2.8 \pm 0.4$ \\
Class E & $2.2 \pm 0.6$ \\
Class F or Central & $1.0 \pm 0.9$ \\
\hline
\end{tabular}

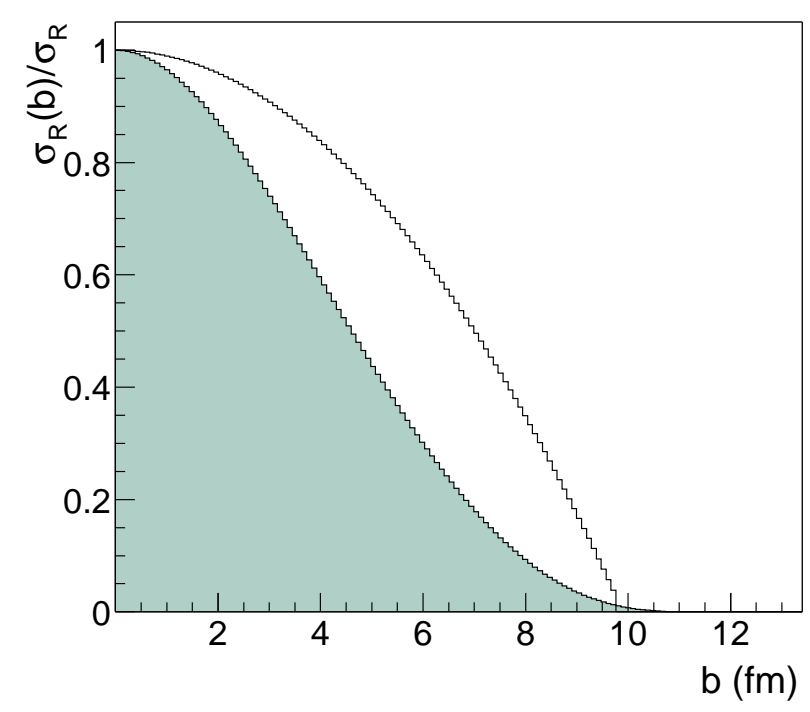

Fig. 4. Evolution of $\sigma_{R}(b) / \sigma_{R}$ as a function of the impact parameter without (empty histogram) and with (filled histogram) the condition that a hard photon is produced.

\subsection{Centrality dependence of the hard photon production}

The two-exponential fit of Eq. 11 reproduces the hard photon energy spectrum of each one of the six centrality classes, from peripheral to central events. The corresponding direct and thermal slopes parameters and intensities are listed in Table 5 . Whereas the measured $E_{0}^{t}(\mathrm{~b})$ differ by up to $24 \%$ from the inclusive value, the direct slope variation amounts to less than $5 \%$ of the inclusive $E_{0}^{d}$. The measurement of an almost constant direct slope confirms that the $E_{0}^{d}$ is an observable that depends just on the bombarding energy [9]. Only for very peripheral reactions it is expected to observe a decrease of the direct photon slope mainly due to the decrease of the Fermi momentum in the surface of the nucleus 31. In Fig. [5 the values of the thermal (direct) slopes are displayed as a function of the thermal (direct) multiplicities. The direct hard photon slope is independent of the direct hard photon multiplicity. This shows that the increase of the direct hard $\gamma$ multiplicity with the centrality is just a consequence of the associated increase of the number of first-chance pn collisions. On the other hand, the thermal slope exhibits a linear dependence on the thermal multiplicity. This trend, observed for the first time, indicates that, assuming that the size of the thermalized source remains almost constant with centrality, the thermal production is sensitive to the excitation of the nuclear system. This result is supported by the inclusive thermal systematics collected by the TAPS Collaboration: the thermal slopes and thermal multiplicities divided by the size of the system, scale with the energy available in the nucleus-nucleus center-of-mass [10.24.

\subsection{Exclusive analysis of the GDR emission}

Exploiting the wide dynamic range of TAPS, we have estimated the $\gamma$ yield from the Giant Dipole Resonance (GDR) decay emitted in peripheral ${ }^{129} \mathrm{Xe}+{ }^{n a t} \mathrm{Sn}$ reactions. The main motivation of this investigation was to identify a possible thermal bremsstrahlung and GDR mixture in the low energy range $\left(E_{\gamma} \approx 30 \mathrm{MeV}\right)$ of the hard photon spectra, which could distort the measurement of the thermal hard photon yield. Peripheral reactions exhibit the highest probability of GDR $\gamma$ contamination because the reached excitation energies can still be not high enough for suppression of the collective mechanisms responsible of the GDR $\gamma$ emission [34, and because of the softer observed thermal emission. So the GDR analysis in peripheral reactions allowed us to conclude about a possible GDR $\gamma$ contamination for all reaction classes.

In order to perform the GDR $\gamma$ analysis, the direct and thermal hard photon components have been subtracted from the total photon spectra. This subtraction is done by extrapolating the double hard $\gamma$ exponential fit down to the lowest energy, given by the TAPS LED threshold of $10 \mathrm{MeV}$. This extrapolation technique is routinely applied in GDR measurements from HI reactions (see e.g. 32,33, 34]). 
R. Ortega et al.: Constraints on the time-scale of nuclear breakup from thermal hard-photon emission

Table 5. Characteristics of the hard photon spectra measured in the six different centrality classes: direct (E $\left.E_{0}^{d}\right)$ and thermal $\left(E_{0}^{t}\right)$ slopes, ratio of thermal to total hard-photon intensities $\left(I_{t} / I_{\text {tot }}\right)$, and thermal photon multiplicity $\left(M_{\gamma}^{t}\right)$.

\begin{tabular}{llllll}
\hline Centrality class & $E_{0}^{d}(\mathrm{MeV})$ & $E_{0}^{t}(\mathrm{MeV})$ & $I_{t} / I_{\text {tot }}(\%)$ & $M_{\gamma}^{t}$ & $\chi^{2} / n d f$ \\
\hline A or Periph. & $14.9 \pm 0.9$ & $5.7 \pm 0.5$ & $19 \pm 1$ & $(7.6 \pm 0.7) \cdot 10^{-5}$ & 1.5 \\
B & $14.6 \pm 0.9$ & $5.7 \pm 0.5$ & $19 \pm 1$ & $(8.9 \pm 1.1) \cdot 10^{-5}$ & 1.2 \\
C & $15.6 \pm 0.9$ & $6.3 \pm 0.6$ & $19 \pm 1$ & $(3.4 \pm 0.4) \cdot 10^{-4}$ & 1.0 \\
D & $16.0 \pm 1.0$ & $7.8 \pm 0.7$ & $25 \pm 1$ & $(7.8 \pm 0.6) \cdot 10^{-4}$ & 1.4 \\
E & $16.1 \pm 1.1$ & $7.9 \pm 0.7$ & $25 \pm 1$ & $(9.4 \pm 0.6) \cdot 10^{-4}$ & 1.2 \\
F or Central & $15.9 \pm 1.0$ & $8.7 \pm 0.8$ & $27 \pm 2$ & $\left(1.4_{-0.3}^{+0.2}\right) \cdot 10^{-3}$ & 1.0 \\
\hline
\end{tabular}

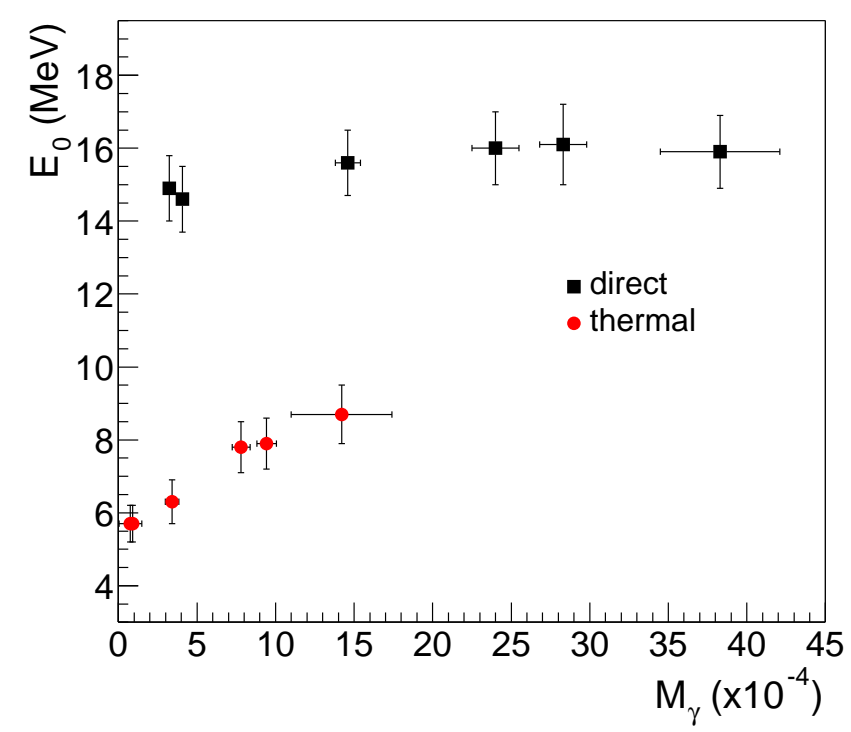

Fig. 5. Direct and thermal hard photon slopes vs their respective multiplicities for the six Xe+Sn centrality classes considered.

We have compared the GDR spectra measured for peripheral centrality classes $A$ and $B$ with the GDR distributions measured with the multidetector MEDEA and reported by $\mathrm{T}$. Suomijärvi et al. 34. In this work, the GDR $\gamma$ emission from hot nuclei of $\mathrm{A} \approx 115$ formed in the ${ }^{36} \mathrm{Ar}+{ }^{90} \mathrm{Zr}$ at $27 \mathrm{~A} \mathrm{MeV}$ reaction is investigated in detail. The comparison of the GDR $\gamma$ yield measured in this analysis and the one measured for peripheral reactions in our work comes easily to mind when considering the similarities in mass and in excitation energy of the emitting sources:

- In peripheral ${ }^{129} \mathrm{Xe}+{ }^{n a t} \mathrm{Sn}$ reactions the quasi-projectile and quasi-target are close to the ${ }^{129} \mathrm{Xe}$ and ${ }^{\text {nat }}$ Sn masses. Assuming that both, quasi-target and quasi-projectile, develop collective GDR oscillations after the collision, the photon spectrum measured in the peripheral centralities scaled by a factor of $\sim 1 / 2$ can be compared to the GDR $\gamma$ yield emitted by a nuclei of $\mathrm{A} \sim 115$;
- In spite of the different geometry and bombarding energy of both reactions, the excitation energies reached at large impact parameters in ${ }^{129} \mathrm{Xe}+{ }^{n a t} \mathrm{Sn}$ reactions ( 3A MeV, see next section) are comparable with the $\epsilon^{\star}$ attained in the ${ }^{36} \mathrm{Ar}+{ }^{90} \mathrm{Zr} \mathrm{MeV}$ reaction at $27 \mathrm{~A}$. Besides, the GDR yield measured for the ${ }^{36} \mathrm{Ar}+{ }^{90} \mathrm{Zr}$ system has been found to remain constant with the excitation energy 34] (see Fig. 6).

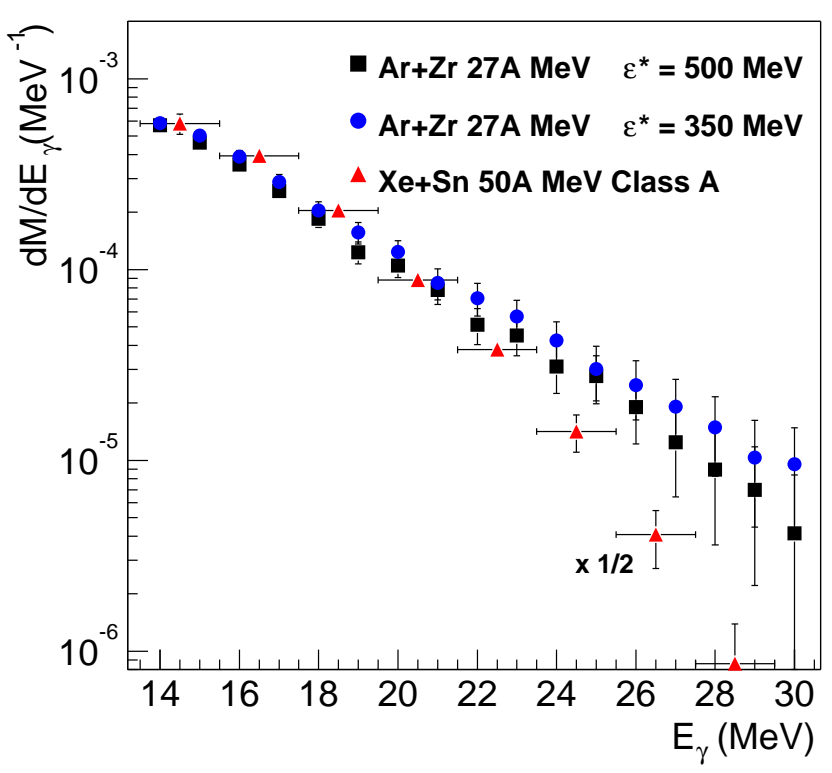

Fig. 6. Spectra in the GDR region for the ${ }^{36} \mathrm{Ar}+{ }^{90} \mathrm{Zr}$ reaction [34], for two different excitation energies: $\epsilon^{\star}=350 \mathrm{MeV}$ (dots), $\epsilon^{\star}=500 \mathrm{MeV}$ (squares)[34], compared to the experimental $\gamma$ spectrum obtained in peripheral ${ }^{129} \mathrm{Xe}+{ }^{\text {nat }} \mathrm{Sn}$ reactions (triangles) after subtraction of the hard-photon yield (extrapolated down to $10 \mathrm{MeV}$ ).

The GDR yields (the $\gamma$ spectra after subtraction of the bremsstrahlung contribution) for the ${ }^{36} \mathrm{Ar}+{ }^{90} \mathrm{Zr}$ reaction for $\epsilon^{\star}=350 \mathrm{MeV}$ and for $\epsilon^{\star}=500 \mathrm{MeV}$ from 34, and the GDR yield estimated for the ${ }^{129} \mathrm{Xe}+{ }^{n a t} \mathrm{Sn}$ centrality class 
R. Ortega et al.: Constraints on the time-scale of nuclear breakup from thermal hard-photon emission

$\mathrm{A}^{1}$ are plotted together in Fig. 6] The global compatibility of the three distributions assures the validity of the estimation of the GDR yield for the peripheral $\mathrm{Xe}+\mathrm{Sn}$ hard photon spectra. The difference found in the $25-30 \mathrm{MeV}$ region between the TAPS and MEDEA measurements is due to the fact that in the latter the subtracted hard photon distribution is parameterized by a single exponential. Taking into account that for peripheral reactions the total photon multiplicity is around $9 \cdot 10^{-5}$ at $E_{\gamma}=30 \mathrm{MeV}$ and that the GDR yield at this energy is found to be lower than $10^{-5}$ (see Fig. 6), we conclude that the GDR contamination in the low energy range of the hard photon spectra can be neglected. This result is in good agreement with the highest energy GDR gamma contribution predicted from the systematics. Indeed, taking the centroid energy of the GDR as $E_{G D R}=76.5 A^{-1 / 3}$ and the maximum width $\Gamma_{G D R} \approx 12 \mathrm{MeV}$ 34 35, the GDR emission neither from the Xe projectile nor from the Sn target are expected to exceed $28 \mathrm{MeV}$.

\section{Derived (thermo)dynamical properties}

\subsection{Nuclear Temperature}

Although, due to their long mean free path in nuclear matter, thermal hard photons are not in thermal equilibrium with the source, their spectral shape is directly correlated to the temperature of the radiating nuclear fragment. As in our previous analyses [12, we use the kinetic thermal model of Neuhauser and Koonin 11] to calculate the relation between the inverse slope $E_{0}^{t}$ and the temperature $T$ of the nuclear system. The electromagnetic radiation rate emitted by a hot and equilibrated nuclear fragment is expressed in this model as a function of the local density and temperature of the source. It is assumed that bremsstrahlung in proton-neutron collisions is the main source of thermal photon emission for $E_{\gamma}>30 \mathrm{MeV}$. The hard photon spectra thus calculated are accurately approximated by an exponential with slope $E_{0}^{t}$ in agreement with the experimental data [12. The evolution of $E_{0}^{t}$ values extracted from a fit above $30 \mathrm{MeV}$ with the temperature, $\mathrm{T}$, is quantitatively well described by the following linear expression:

$$
T(\mathrm{MeV})=(0.78 \pm 0.02) \cdot E_{0}^{t}(\mathrm{MeV})
$$

in the region $\mathrm{T} \approx 3-10 \mathrm{MeV}$ and $\rho \approx(0.3-1.2) \rho_{0}$ [12]. We have applied this photon thermometer to extract the temperature of the radiating system produced in the different ${ }^{129} \mathrm{Xe}+{ }^{n a t} \mathrm{Sn}$ centralities studied in this work. The obtained temperatures are reported in Table 6. The temperature of the nuclear system for central collisions (centrality class $\left.\mathrm{F}, b / b_{\max } \leq 0.1\right)$ is a $50 \% \pm 20 \%$ higher than for peripheral collisions (centrality class $\mathrm{A}, b / b_{\max } \approx 0.6$ ).

\footnotetext{
1 the GDR yield measured for centrality classes A and B are almost identical, so only the yield for class $\mathrm{A}$ is shown in the figure.
}

Table 6. Nuclear temperatures T estimated through Eq. 4 from the experimental thermal hard photon slopes $E_{0}^{t}$ of the inclusive and exclusive hard photon spectra measured in ${ }^{129} \mathrm{Xe}+{ }^{\text {nat }} \mathrm{Sn}$ at $50 \mathrm{~A} \mathrm{MeV}$.

\begin{tabular}{lll}
\hline Centrality class & $E_{0}^{t}(\mathrm{MeV})$ & $\mathrm{T}(\mathrm{MeV})$ \\
\hline Inclusive & $7.0 \pm 0.6$ & $5.5 \pm 0.8$ \\
A or Peripheral & $5.7 \pm 0.5$ & $4.4 \pm 0.4$ \\
B & $5.7 \pm 0.5$ & $4.4 \pm 0.4$ \\
C & $6.3 \pm 0.6$ & $4.9 \pm 0.5$ \\
D & $7.8 \pm 0.7$ & $6.1 \pm 0.5$ \\
E & $7.9 \pm 0.7$ & $6.2 \pm 0.5$ \\
F or Central & $8.7 \pm 0.8$ & $6.8 \pm 0.6$ \\
\hline
\end{tabular}

\subsection{Lifetime of the thermalized system}

The multiplicity of hard photons emitted by an equilibrated nuclear fragment can be calculated by means of the model of Neuhauser and Koonin from the following expression [12]:

$$
M_{\gamma}^{N K}=\int d^{3} x \int d t \int_{30}^{\infty} \frac{d R_{\gamma}^{N K}(T, \rho)}{d E_{\gamma}} d E_{\gamma}
$$

where $R_{\gamma}^{N K}(T, \rho)$ is the rate of photons emitted from a thermal nuclear fragment with temperature $T$ and density $\rho$. From this model, $R_{\gamma}^{N K}(T, \rho)$ is found to scale with $\sim T^{6.7}$ and to be proportional to $\rho$. The quadratic dependence of the photon rate on $\rho$ expected naively turns out to be linear due to the Pauli blocking. The photon rate can be hence expressed as $R_{\gamma}^{N K} \approx R_{0}^{N K} \cdot T^{6.7} \cdot \rho$, with the constant $R_{0}^{N K}=1.1 \cdot 10^{-13} \mathrm{MeV}^{-6.7} \cdot(\mathrm{fm} / c)^{-1}$. We can further simplify Eq. $[5$ by assuming that the temperature is uniform in the volume $V$ and roughly constant during the emission time,

$$
M_{\gamma}^{N K} \approx V \cdot \Delta \tau \cdot R_{0}^{N K} \cdot T^{6.7} \cdot \rho
$$

where $V$ and $\Delta \tau$ are respectively the volume and the lifetime of the radiating nuclear source. The volume can be approximated as the ratio of the sum and the projectile and target nucleons ${ }^{2}$ over the nuclear density, $V \approx\left(\mathrm{A}_{t}+\mathrm{A}_{p}\right) / \rho$.

With these simplifying assumptions, the thermal hard photon yield, scaled to the relative size of the system, can be written as:

$$
\frac{M_{\gamma}^{N K}}{\left(A_{t}+A_{p}\right)} \approx \Delta \tau \cdot R_{0}^{N K} \cdot T^{6.7}
$$

This relation, which is independent of the density, can be exploited to estimate the lifetime $\Delta \tau$ of the thermal equilibrated source, by fitting Eq. (7) to the experimentally measured multiplicities (reported in Table 5) as a function

\footnotetext{
${ }^{2}$ We neglect here emission of particles during preequilibrium.
} 
of the temperature. Figure 7 shows the experimental (inclusive) $M_{\gamma}^{t}$, divided by size of the system $A_{t o t}=A_{t}+A_{p}$, as a function of the estimated $T$, for the $\mathrm{Xe}+\mathrm{Sn}$ and the six other reactions studied [12. The lines correspond to the calculated $M_{\gamma}^{N K}$ as a function of the $T$ for two different lifetimes $(\Delta \tau=100 \mathrm{fm} / c$ and $\Delta \tau=35 \mathrm{fm} / c)$.

The calculated multiplicities successfully reproduce the experimental thermal hard photon yields measured for all the reactions (see Figs. [7] and [8), with $\Delta \tau$ values about $100 \mathrm{fm} / c$, which are consistent with the expected lifetime of an equilibrated source (see e.g. [36]). This result con-

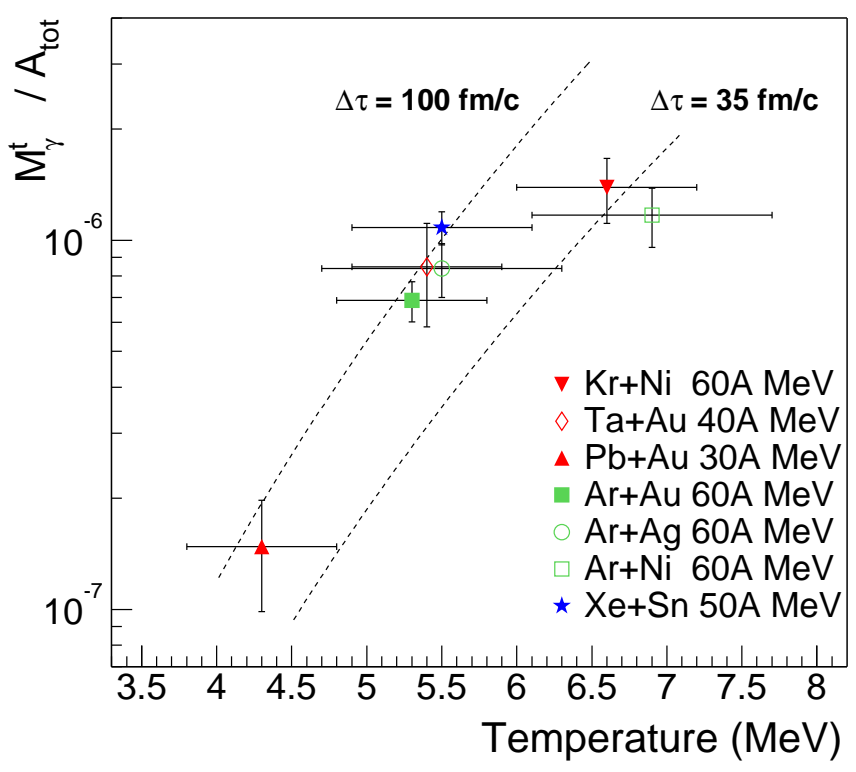

Fig. 7. Experimental thermal bremsstrahlung multiplicity, $M_{\gamma}^{t}$, divided by the size of the colliding system, $A_{\text {tot }}=A_{t}+A_{p}$, plotted as a function of the extracted nuclear temperatures, for the different reactions studied by the TAPS Collaboration. The lines correspond to two different values of the source lifetime estimated with Eq. 7

firms the validity of the thermal model, since it reproduces both experimental observables, the slope of the thermal hard photon spectrum and the thermal multiplicity, with the temperature as the single input parameter. In Figs. 7 and 8 it can be observed that all the systems studied have a lifetime $\Delta \tau$ of the order or larger than $100 \mathrm{fm} / c$, except the two lighter systems at $60 \mathrm{~A} \mathrm{MeV},{ }^{86} \mathrm{Kr}+{ }^{58} \mathrm{Ni}$ and ${ }^{36} \mathrm{Ar}+{ }^{58} \mathrm{Ni}$, which seem to survive a shorter $\Delta \tau \sim 35$ $\mathrm{fm} / c$, similar to the transit time of the colliding ions. In these two reactions, the achieved excitation energy is also higher than in the rest of reactions 12. This difference in $\Delta \tau$ might indicate that equilibrated nuclear sources break faster when they have a smaller size or higher excitation energies, as observed in [36 37/38]. The experimental thermal hard photon multiplicities measured for the ${ }^{129} \mathrm{Xe}+$

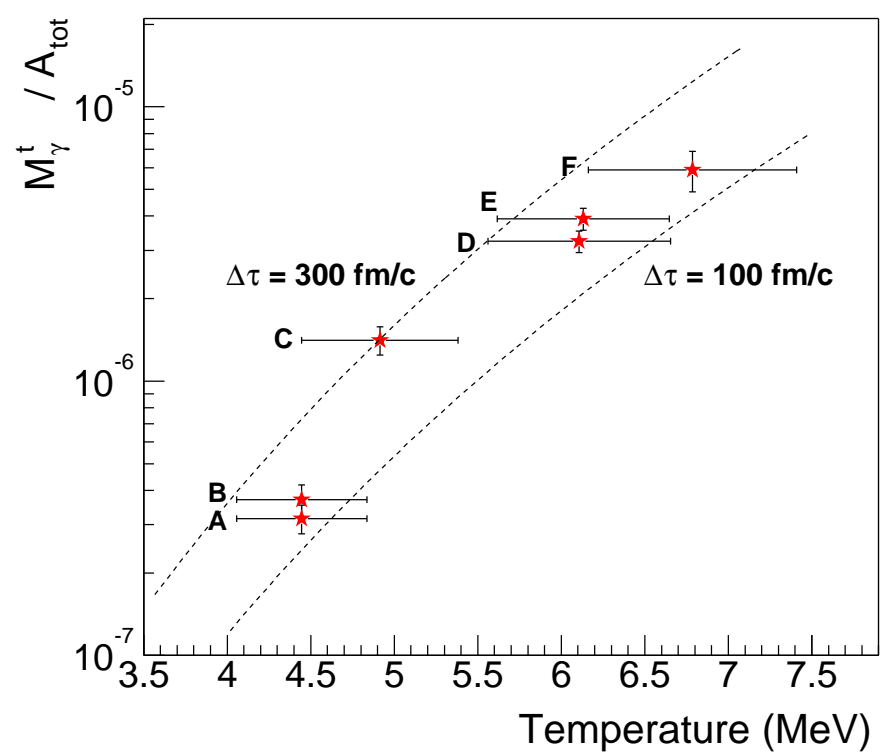

Fig. 8. Thermal hard photon multiplicities, $M_{\gamma}^{t}$, divided by the total size of the system, $A_{\text {tot }}=A_{t}+A_{p}$, plotted as a function of the nuclear temperature extracted from Eq. 4 for the different ${ }^{129} \mathrm{Xe}+{ }^{\text {nat }} \mathrm{Sn}$ centrality classes. The lines correspond to two different values of source lifetime computed with Eq. 7 .

${ }^{n a t}$ Sn centrality classes exhibit a trend compatible with lifetimes of the order of 100-300 fm/c (Fig 8). In our measurements, within the data uncertainty, we do not observe any change of the lifetime of the equilibrated fragment with the impact parameter of the collision for $\mathrm{Xe}+\mathrm{Sn}$. The relatively large lifetime values obtained, jointly with the measurement of an important production of thermal hard photons, suggest that, at least for the investigated $\mathrm{Xe}+\mathrm{Sn}$ reactions, the fireball does not undergo an instantaneous breakup from a rapidly expanding state (spinodal decomposition).

\subsection{Caloric curve constructed from $\mathrm{Xe}+\mathrm{Sn}$ exclusive measurements}

As done in a previous paper for inclusive thermal photon measurements [12, we have measured the relationship between the estimated temperature and the excitation energy, $\epsilon^{\star}$, of the hot equilibrated fragment formed in $\mathrm{Xe}+\mathrm{Sn}$ reactions at the different centralities studied in this work. At variance with experiments that measure the excitation energy with charged particle detectors, we cannot determine "calorimetrically" $\epsilon^{\star}$ from our data. The excitation energy of Xe quasiprojectiles produced from peripheral to central collisions has been deduced by Steckmeyer et al. [18] as a function of the charged particle multiplicity measured by the INDRA detector. The methods described in [18] are based on the determination of the 
velocity of the quasiprojectile. The excitation energy is calculated from the kinetic energies of products belonging to the source. We have employed the geometric method of Cavata et al. 29] to relate the average impact parameter of each $\mathrm{Xe}+\mathrm{Sn}$ reaction class with the corresponding value of $M_{c p}^{I N D R A}$, in order to obtain the measured value of $\epsilon^{\star}$ for each centrality class. The estimated $M_{c p}^{I N D R A}$ and $\epsilon^{\star}$ values are reported in Table 7

Table 7. Values of the charged particle multiplicity measured with INDRA, $M_{c p}^{I N D R A}$, and of the estimated excitation energy, $\epsilon^{\star}$, for each Xe+Sn centrality class with averaged impact parameter $\langle b\rangle$.

\begin{tabular}{llll}
\hline Centrality class & $\langle\mathrm{b}\rangle(\mathrm{fm})$ & $M_{c p}^{I N D R A}$ & $\epsilon^{\star}(A \mathrm{MeV})$ \\
\hline Inclusive & $3.8 \pm 1.0$ & $25 \pm 1$ & $5.6 \pm 1.0$ \\
A or Peripheral & $7.6 \pm 0.3$ & $14 \pm 1$ & $2.8 \pm 0.3$ \\
B & $7.2 \pm 0.3$ & $16 \pm 1$ & $3.3 \pm 0.4$ \\
C & $4.2 \pm 0.4$ & $24 \pm 1$ & $5.2 \pm 0.6$ \\
D & $2.8 \pm 0.4$ & $27 \pm 1$ & $6.1 \pm 0.7$ \\
E & $2.2 \pm 0.6$ & $29_{-1}^{+2}$ & $6.7 \pm 0.7$ \\
F or Central & $1.0 \pm 0.9$ & $32_{-2}^{+5}$ & $7.7_{-1.0}^{+1.4}(8.7 \pm 1.5)$ \\
\hline
\end{tabular}

The errors of $\epsilon^{\star}$ take into account the uncertainty of $\langle b\rangle$ and $\mathrm{a} \sim 10 \%$ systematic error due to the reconstruction of the quasiprojectile 18. As expected, the excitation energy decreases linearly with increasing impact parameter. Since the highest measured charged particle multiplicity does not correspond to a real $\langle b\rangle=0$, the geometrical method used to relate $\langle\mathrm{b}\rangle$ and $M_{c p}^{I N D R A}$ is not accurate enough for central collisions. We therefore also quote in parentheses for centrality class $\mathrm{F}$ the value of $\epsilon^{\star}$ predicted for central collisions by the model of Natowitz et al. 39. The caloric curve obtained is displayed in Fig. 9 The temperature increases smoothly with the excitation energy, the $\left(\epsilon^{\star}, \mathrm{T}\right)$ pairs obtained are below the expected Fermi fluid curves $\left(T=\sqrt{K \epsilon^{\star}}(\mathrm{MeV})\right.$ with $\mathrm{K}=8 / \mathrm{A}-13 / \mathrm{A} \mathrm{MeV}$ covering the known range of average nuclear level density parameters [0]). Peripheral reactions (classes $\mathrm{A}$ and $\mathrm{B}$ ) lead to excitation energies $\epsilon^{\star} \approx 3 A \mathrm{MeV}$, near those identified as the onset of the leveling region of the caloric curve for nuclear systems of size $\mathrm{A}=180-241$ [4].

\section{Summary}

Hard photon $\left(E_{\gamma}>30 \mathrm{MeV}\right)$ emission has been studied in ${ }^{129} \mathrm{Xe}+{ }^{n a t} \mathrm{Sn}$ at $50 \mathrm{~A} \mathrm{MeV}$ for six bins of impactparameter. The thermal component has been determined from a double source analysis of the measured spectra. For each centrality class, the temperature of the produced nuclear systems has been extracted from the measured thermal hard photon slope, by applying a thermal nuclear bremsstrahlung model that successfully reproduces the measured thermal hard photon spectral shape and multiplicities. The temperature obtained exhibits a small

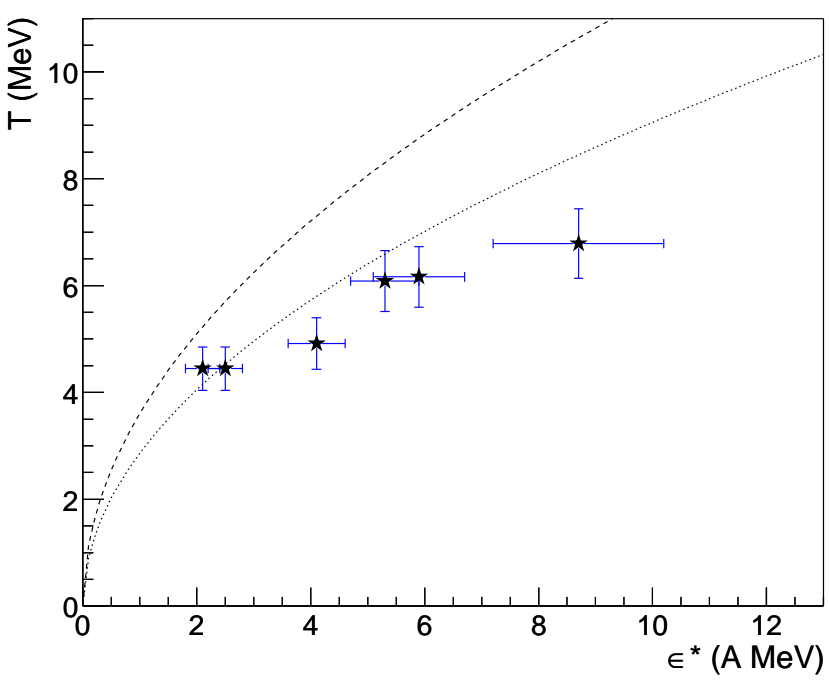

Fig. 9. Caloric curve constructed from the thermal hard photon slope measurements for the ${ }^{129} \mathrm{Xe}+{ }^{\text {nat }} \mathrm{Sn}$ reaction at $50 \mathrm{~A}$ MeV (Table 6) and the estimated excitation energies (Table 7), compared to the Fermi liquid model curves with inverse leveldensity parameters $K=8 / A \mathrm{MeV}$ (dashed line) and $K=13 / \mathrm{A}$ $\mathrm{MeV}$ (dotted line).

but systematic increase with decreasing impact parameter, from $T=4.4 \mathrm{MeV}$ for the most peripheral collisions $\left(b / b_{\max } \approx 0.6\right)$ to $T=6.8 \mathrm{MeV}$ for the most central class $\left(b / b_{\max } \leq 0.1\right)$. The amount of second-chance bremsstrahlung emission measured in each centrality bin is well described by the thermal model by assuming emission times of the order $\tau \approx 100-300 \mathrm{fm} / c$ for all centralities. The lifetimes of the produced equilibrated sources have also been estimated for six other heavy-ion reactions for which thermal bremsstrahlung emission has been measured by the TAPS Collaboration. The inclusive thermal hard photon multiplicities measured as a function of the temperature $T$ for the different reactions suggest lifetimes $\tau \sim 100 \mathrm{fm} / c$, i.e. between two and three times longer than the transit time of the colliding ions at these energies. Such relatively large time scale disfavor scenarios of instantaneous spinodal break-up and is more consistent with sequential fragmentation from a thermally equilibrated source. It is worth noting, however, that for the two smallest target-projectile combinations, ${ }^{86} \mathrm{Kr}+{ }^{58} \mathrm{Ni}$ and ${ }^{36} \mathrm{Ar}+{ }^{58} \mathrm{Ni}$ at $60 \mathrm{~A} \mathrm{MeV}$, which are the systems with more energy available in the nucleus-nucleus center-of-mass, the experimental thermal photon multiplicities seem to be better reproduced with shorter lived equilibrated sources $(\tau \sim$ $35 \mathrm{fm} / c)$.

Finally, we present for the first time a caloric curve, $\epsilon^{\star}(T)$, obtained from thermal hard photon measurements for different impact parameter bins of the same reaction, $\mathrm{Xe}+\mathrm{Sn}$. Such a curve falls below the one expected for a Fermi liquid, in agreement with the curve constructed from thermal hard photon slopes measured in different 
R. Ortega et al.: Constraints on the time-scale of nuclear breakup from thermal hard-photon emission

heavy-ion reactions [12, and consistent also with the available systematics of nuclear caloric curves [4.

\section{Acknowledgements}

We thank the GANIL accelerator staff for providing a high quality beam and technical support during the experiment. We thank J.C. Steckmeyer and the INDRA Collaboration for providing the charged-particle multiplicity data, and the MEDEA Collaboration for providing the GDR data. This work has been in part supported by IN2P3-CICYT PN97-1 agreement, by the Dutch Foundation FOM, by the European Union HCM network under Contract No. HRXCT94066, and by DGICYT under contract FPA2000-2041-C02-01. R. Ortega aknowledges support given by the Alexander von Humboldt Foundation. D.d'E. acknowledges support by the European Union TMR Programme (Marie-Curie Fellowship No. HPMFCT-1999-00311).

\section{References}

1. J. P. Bondorf, A.S. Botvina, A.S. Ilinov, I.N. Mishustin and K. Sneppen, Phys. Rept. 257 (1995) 133; L.G. Moretto and G.J. Wozniak, Ann. Rev. Nucl. Part. Sci. 43 (1993) 379.

2. V. E. Viola, Nucl. Phys. A 734 (2004) 487.

3. J. Pochodzalla et al., Phys. Rev. Lett. 75, 1040 (1995).

4. J. B. Natowitz, Phys. Rev. C65, 034618 (2002).

5. J. B. Natowitz et al., Phys. Rev. Lett. 89, 212701 (2002).

6. W. Trautmann, nucl-ex/0411023 Proceedings INPC2004, Goeteborg, Sweden, June 27-July 2 (2004).

7. H. Nifenecker and J. Pinston, Ann. Rev. Nucl. Part. Sci. 40, 113 (1990).

8. G. Martínez et al., Phys. Lett. B349, 23 (1995).

9. Y. Schutz et al., Nucl. Phys. A622, 404 (1997).

10. D. d'Enterria et al., Phys. Rev. Lett. 87, 22701 (2001).

11. D. Neuhauser and S.E. Koonin, Nucl. Phys. A462, 163 (1987).

12. D. d'Enterria et al., Phys. Lett. B538, 27 (2002).

13. M.J. van Goethem et al., Phys. Rev. Lett. 88, 122302 (2002).

14. N. Marie Phys. Lett. B391, 15 (1997).

15. N. Marie et al., Phys. Rev. C58, 256 (1999).

16. D. Gourio et al., Eur. Phys. J. A7, 245 (2000).

17. N. Bellaize et al., Nucl. Phys. A709, 367 (2002).

18. J.C. Steckmeyer et al., Nucl. Phys. A686, 537 (2001).

19. R. Novotny, IEEE Trans. Nucl. Sci. 38, 379 (1991).

20. M. Laval et al., Nucl. Inst. and Meth. A206, 69 (1983).

21. A. Gabler et al., Nucl. Inst. and Meth. A346, 164 (1994).

22. D.W. Stracener et al., Nucl. Inst. and Meth. 294, 485 (1990).

23. S. Luke et al., Phys. Rev. C47, 1211 (1993).

24. R. Ortega, Ph.D.Thesis, Univ. Aut. de Barcelona, 2003.

25. L. Tassan-Got, Nucl. Inst. Meth. B194, 503 (2002).

26. J. van Pol, Ph.D.Thesis, Rijkuniversiteit Groningen, 1995.

27. R. Ortega, F. Fernández, D. D'Enterria and G. Martínez,

Acta Phys. Polon. B 33, 965 (2002).

28. H. Nifenecker and J.P. Bondorf Nucl. Phys. A442, 478

(1985).
29. C. Cavata et al., Phys. Rev. C294, 47 (1993).

30. S. Riess et al., Phys. Rev. Lett. 69, 1504 (1992).

31. G. Martínez et al., Phys. Lett. B334, 23 (1994).

32. J.J. Gaardhoje et al., Phys. Rev. Lett. 59, 1409 (1987).

33. J.H. Le Faou et al., Phys. Rev. Lett. 74, 3321 (1994).

34. T. Suomijärvi et al., Phys. Rev. C53, 2258 (1996).

35. B.L. Berman and S.C. Fultz, Rev. Mod. Phys. 47, 713 (1975).

36. R. Wada et al., Phys. Rev. C69, 044610 (2004).

37. L. Beaulieu et al., Phys. Rev. C84, 5974 (2000).

38. Z. He et al., Phys. Rev.C63, 011601 (2001).

39. J.B. Natowitz et al., Z. Phys. A325, 467 (1986).

40. B. Nerlo-Pomorska et al, Int. J. Mod. Phys. E 14 (2005) 505 